diagnosed using the Diagnostic and Statistical Manual of Mental Disorders (DSM-IV) criteria.

Results People with PTSD were more likely than those without PTSD to have hypertension even after adjusting for age, sex, world region of origin, length of stay in the Netherlands, and obesity $(\mathrm{OR}=1.72,95 \%$ CI 1.50 to 1.96$)$. There was an interaction between PTSD and co-morbid depression $(p=0.006)$, indicating that the effect of PTSD and depression on hypertension were independent. When the analyses were stratified by depression status, among nondepressed group, individuals with PTSD had higher odds of hypertension than those without PTSD (OR=1.36, 95\% CI 1.17 to 1.59 ). However, among the depressed individuals, there was no association between PTSD and hypertension even after adjustment for other factors: 1.09 (95\% CI 0.77 to 1.53 ).

Conclusions Our findings suggest that there is a positive relationship between PTSD and hypertension among asylum seekers in the Netherlands; and this relationship is independent of comorbid depression. Clinicians and policy makers need to take history of PTSD into account when screening and treating asylum seekers for hypertension.

\section{P2-101 PRISONER MORTALITY IN SCOTLAND 1996-2007: RETROSPECTIVE COHORT STUDY}

doi:10.1136/jech.2011.142976i.36

\author{
${ }^{1,2} \mathrm{~L}$ Graham, ${ }^{*} \mathrm{C}$ Fischbacher, ${ }^{1} \mathrm{D}$ Stockton, ${ }^{2} \mathrm{~A}$ Fraser, ${ }^{1} \mathrm{M}$ Fleming, ${ }^{1} \mathrm{~K}$ Grieg. ${ }^{1}$ Infor-
} mation Services Division, Edinburgh, UK; ${ }^{2}$ Scottish Prison Service, Edinburgh, UK

Introduction Mortality is substantially increased among those who have been imprisoned but there is little information about the extent to which excess mortality might be explained by deprivation. All cause mortality among Scottish prisoners has not previously been described.

Methods Standard record linkage methods were used to link Scottish Prison Service and mortality data for individuals imprisoned in Scotland for the first time between 1 January 1996 and 31 December 2007.

Results Among 76627 individuals there were 4414 deaths (3928 in men). Compared to the general population the RR of mortality (adjusted for age and year of death) was 3.3 (95\% CI 3.2 to 3.4 ) for men and 7.5 (6.8 to 8.2) for women. Further adjustment for deprivation accounted for part of this excess (adjusted risks $2.3(2.2,2.4)$ and $5.6(5.1,6.1)$ for men and women respectively). RRs were highest for drug and alcohol related causes, suicide and homicide and were markedly higher among women than men for these causes. Out of prison death rates were highest in the first week after discharge from prison. Mortality rates were lower in those with longer total duration in prison and higher in those with more episodes in prison.

Conclusions People who have been imprisoned in Scotland experience substantial excess mortality that is only partly explained by their levels of deprivation. The association of increased mortality with multiple shorter periods in prison and the concentration of deaths in the early period after prison discharge have substantial implications for policy and practice.

\section{P2-102 DEVELOPMENTAL TRAJECTORIES OF BODY MASS INDEX IN CHILDHOOD: IS MATERNAL SMOKING DURING PREGNANCY A CRITICAL EXPOSURE?}

doi:10.1136/jech.2011.142976i.37

\begin{abstract}
1,2 J Gravel, * ${ }^{2} \mathrm{~B}$ Potter, ${ }^{1,2} \mathrm{~L}$ Dubois. ${ }^{1}$ Institute of Population Health, University of Ottawa, Ottawa, Ontario, Canada; ${ }^{2}$ Department of Epidemiology and Community, University of Ottawa, Ottawa, Ontario, Canada
\end{abstract}

Introduction Intrauterine life has been identified as a critical period for the development of obesity. Research has consistently shown that prenatal exposure to maternal cigarette smoke (PEMCS) is associated with a number of adverse fetal, obstetrical and developmental outcomes. While PEMCS has emerged as an important risk factor for overweight in offspring, no consensus exists on the pattern or duration of impact. This study seeks to further examine the role of PEMCS on developmental body mass index (BMI) trajectories in children up to 10 years of age.

Methods Data on mother-child pairs $(n=1291)$ was obtained from the Quebec Longitudinal Study of Child Development, which includes a range of social and biological information on child development. Developmental BMI trajectories were established empirically, with a group based modelling strategy, using repeated measurements of height of weight up to age 10. This approach (TRAJ) complements both hierarchical and latent growth curve modelling for analysing developmental trajectories.

Results Four distinct BMI trajectories were identified: early onset overweight $(4.4 \%)$, increasing to overweight $(2.7 \%)$, stable overweight $(26.1 \%)$ and never overweight $(66.8 \%)$. PEMCS was associated with increased risk of overweight at age 10 (OR: 3.3, 95\% CI 1.96 to 5.57$)$ and with trajectory membership ( $<<0.003)$.

Conclusions The elevated risk of excess weight among the offspring of smoking mothers supports the paradigm of in utero and early life obesity prevention. Our approach to longitudinal childhood weight analysis, which allows for heterogeneous population trajectories, may help to improve our understanding of the different pathways leading to obesity in adulthood.

\section{P2-103 IS THE SOCIOECONOMIC PATTERNING OF TYPE II DIABETES MEDIATED BY SLEEPING PROBLEMS? EVIDENCE FROM THE WEST OF SCOTLAND}

doi:10.1136/jech.2011.142976i.38

${ }^{1} \mathrm{M}$ Green, ${ }^{* 1} \mathrm{~T}$ Robertson, ${ }^{2} \mathrm{C}$ Espie, ${ }^{1} \mathrm{M}$ Benzeval. ${ }^{1} \mathrm{MRC} / \mathrm{CSO}$ : Social and Public Health Sciences Unit, Glasgow, UK; ${ }^{2}$ University of Glasgow Sleep Centre, Glasgow, UK

Introduction Sleep deprivation produces physiological changes similar to those that occur in the development of diabetes. Sleeping patterns may therefore act as a mediator between socioeconomic status and diabetes.

Methods Data are from the West of Scotland Twenty-07 Study. 953 respondents aged approximately 35 at baseline and followed for 20 years with four repeat interviews had valid data and were free of diabetes at baseline. Diabetes at age 55 was identified by self-report of diabetes, diabetic medication or $\mathrm{HbA1c}$ values 26.2. Latent class analysis identified four common patterns of sleep over the 20 years: healthy sleep, problems maintaining sleep, developing problems, and chronic sleeping problems. These were used in a path analysis to investigate whether social class differences in diabetes incidence were mediated by sleeping patterns, adjusting for baseline smoking status, BMI and gender.

Results People in a manual social class at baseline were 2.3 times more likely to have diabetes at age 55 compared to those from nonmanual classes (95\% CIs: 1.32 to 3.99), controlling for baseline characteristics. With further adjustment for long-term sleeping patterns this OR was reduced to 2.03 (95\% CI 1.28 to 3.22). Those from a manual social class were more likely to experience chronic sleeping problems than those from non-manual classes (OR: 2.68, $95 \%$ CI 1.51 to 4.76 ) and those with chronic sleeping problems were more likely to develop diabetes than those with healthy sleeping patterns (OR: 4.22, 95\% CI 1.99 to 8.96 ).

Conclusion Findings support partial mediation of socioeconomic differences in diabetes incidence via long-term sleeping problems. 